\title{
Title: Investigating the Nasal Cycle Using Unilateral Peak Nasal Inspiratory Flow and Acoustic Rhinometry Minimal Cross-Sectional Area Measurements
}

Running Title: A cross-sectional study on prediction of the nasal cycle

$M$ Tan BSc ${ }^{1,3}$, K L Whitcroft MBChB (Hons) BSc MRCS DOHNS ${ }^{1,2}$, N Mehta FRCS ${ }^{1,2}$, A Schilder PhD FRCS ${ }^{1,2}$, T S Leung PhD ${ }^{3}$, P Andrews FRCS ${ }^{1,2}$

1. Department of Rhinology and Facial Plastic Surgery, Royal National Throat Nose and Ear Hospital, London, UK
2. UCL Ear Institute, London, UK
3. Department of Medical Physics and Biomedical Engineering, University College London, UK

Funding: Engineering and Physical Sciences Research Council

Conflict of Interests: None

The corresponding author is Martin Tan. Contact details are:

1. Phone: +44-754-236-2459

2. Email-Address: martin.tan.14@ucl.ac.uk

3. Address: Department of Medical Physics and Biomedical Engineering, University College London, Gower Street, London, WC1E 6BT, United Kingdom

Acknowledgements: C H Li 


\section{Abstract}

\section{Objective}

To plot the nasal cycle using unilateral peak nasal inspiratory flow (UPNIF) and unilateral minimal cross-sectional area (UMCA) readings demonstrating a linear relationship in normal nasal function. Additionally, to determine how this changes in abnormal nasal function.

\section{Design}

A cross-sectional study measuring UPNIF and UMCA in controls demonstrating normal nasal function and in patients with nasal obstruction.

\section{Setting}

Royal National Throat Nose and Ear Hospital, London

\section{Participants}

39 participants, 26 controls and 13 patients, were recruited. Controls exhibited normal nasal function with SNOT-22 $<5$. Patients nasal obstruction symptoms secondary to inflammation or structural abnormality with SNOT-22 $>9$.

\section{Main Outcome Measures and Results}

Airflow rates and resistance values were derived from UPNIF and UMCA measurements respectively based on Poiseuille's laws. Ratios between right and left UPNIF and UMCA values were taken to adjust for confounding factors. The relationship of 1/Resistance Ratio and Airflow Rate Ratio demonstrated a linear of direct proportionality of strong correlation and statistical significance (correlation coefficient $=0.76, p<<0.01$ ). This suggests that data points from controls with a normal nasal cycle lie closely along the regressed line, while those lying significantly away were shown to belong to patients with nasal dysfunction. Olfactory dysfunction appears to be a sensitive discriminator in predicting this.

\section{Conclusion}

This study demonstrates the directly proportional relationship of $1 /$ Resistance Ratio and Airflow Rate Ratio in normal nasal function. Furthermore, nasal pathology can be predicted if data points lie significantly outside these normal limits. Further studies are needed to validate exact normal and abnormal thresholds.

\section{Key Words}

Rhinoplasty, Nasal Septum, Rhinitis, Nose Deformities, Nasal Obstruction, Nasal Cycle 
Level of Evidence

$2 b$ 


\section{INTRODUCTION}

\section{Background}

First described by Kayser[1], the nasal cycle is a physiological phenomenon resulting in the alternating congestion and decongestion of the nasal mucosa. Though not fully understood, the nasal cycle is thought to enable nasal airway resistance regulation, improve nasal defense mechanisms during infection and to allow for unilateral nasal rest. The prevalence of the nasal cycle has been reported to be as high as $80 \%$ in the adult population[2].

Measuring the nasal cycle requires both nasal passages in individuals with normal nasal function to be assessed and then longitudinally repeated. This has been effectively performed using various methods. In particular, unilateral peak nasal inspiratory flow (UPNIF) demonstrates a reasonable correlation with unilateral nasal airway resistance readings using anterior active rhinomanometry (AAR)[3]. This has also been effectively demonstrated using unilateral minimal cross-sectional area (UMCA) and unilateral nasal volume measurements performed by acoustic rhinometry (AR)[4].

However, the ability to assess the nasal cycle at a single time point has not been evaluated. Equally, the ability to determine whether nasal congestion is caused by either the nasal cycle, nasal inflammation or a structural deviation, or a combination of all three remains unknown. Although nasal decongestants can eliminate the nasal cycle and neutralise nasal congestion, which consequently helps delineate a structural cause for nasal obstruction, they cannot differentiate between physiological (e.g. nasal cycle) or pathological (e.g. allergic rhinitis or CRS) causes of nasal congestion[5].

Our primary aim is to determine whether a linear relationship exists between static measurements of UPNIF and UMCA in participants with normal nasal function, based on Poiseuille's Law. This has been previously used to describe bilateral nasal airflow relationships[6,7].

Our secondary aim is to determine if we can differentiate between physiological and pathological cause of nasal congestion. It is known that congestion can cause chaotic changes to airflow and pressure, resulting in air turbulence[8]. Under such circumstances, air no longer flows in parallel layers undisrupted. Since Poiseuille's Law assumes non-turbulent airflow, we expect deviation from the model to occur. We hypothesise that we can determine if this nasal pathology is physiological or pathological based on the extent of air turbulence caused by the nasal pathology $[9,10]$. The larger the deviation from the model due to greater turbulence, the more likely it is pathological and vice versa. The ability to differentiate between a physiological and pathological cause of nasal congestion would be clinically advantageous. 


\section{MATERIALS AND METHODS}

\section{Ethical Considerations}

Full ethical approval was obtained'. Written consent was obtained from all participants.

\section{Study design and Setting}

At the Royal National Throat Nose and Ear Hospital, London, 39 participants, 26 controls and 13 patients, were recruited for this cross-sectional study from February to September $2016^{i i}$.

\section{Subject selection}

Control inclusion criteria: 18 years or above, asymptomatic with regards to any known rhinological condition and a Sino-Nasal Outcome Test 22 (SNOT-22) score of <5; normal olfaction as determined by the "Sniffin' Sticks" test, a normal endoscopic examination by the senior author and a negative skin prick test.

Patient inclusion criteria: symptoms of nasal obstruction, clinical signs of septal deviation, nasal valve collapse or allergic rhinitis or a combination of both with allergic rhinitis confirmed by a skin prick test, and SNOT-22 >9.

Basic demographics such as gender, age, ethnicity, weight and height were noted and were heterogeneous.

\section{Study Protocol}

UPNIF was measured using a modified Youlten (Clement Clark International, UK) flow meter. Participants were instructed to perform the procedure using a standardised protocol and the best of three measurements was used for each side.

An A1 Acoustic Rhinometer (GM Instruments, Kilwinning, United Kingdom) was used. Three consecutive readings of the cross-sectional area of the left and right nasal cavity were taken over a range of $12 \mathrm{~cm} . \mathrm{MCA}_{1}$ (cross-sectional area at the internal nasal valve) and $\mathrm{MCA}_{2}$ (cross-sectional area at anterior half of inferior turbinate), their standard deviations, as well as distance to $M C A_{1}\left(D-M C A_{1}\right)$ and distance to $M C A_{2}\left(D-M C A_{2}\right)$ were automatically calculated and displayed. To simplify analysis, only $\mathrm{MCA}_{1}$ was noted which is the narrowest point in the entire nasal cavity and contributes most greatly to the nasal airway resistance[11,12]. Hence, $\mathrm{MCA}_{1}$ was the sole indicator of resistance.

\footnotetext{
i London - City \& East Research Ethics Committee. Reference: 15/LO/0187).

ii As the participants were selected from a larger clinical study assessing a novel medical device, this section will focus only on data relevant to this publication.
} 
In addition, olfaction was assessed using the 16-item Odour Identification Test as part of the "Sniffin'Sticks" test and allergy was assessed using skin prick tests.

\section{Data Analysis}

Based on Poiseuille's Law, we derived that the resistance is inversely proportional to the square of the cross-sectional area. The following shows how we derived resistance is inversely proportional to the square of the cross-sectional area:

An expression of the nasal airway resistance and factors that affect it can be obtained as shown in equation 1:

$$
R=\frac{8 \mu L}{\pi r^{4}}
$$

where: $\mathrm{R}=$ resistance

$\Delta \mathbf{P}=$ driving pressure

$Q=$ flow rate

$\boldsymbol{\mu}=$ viscosity of fluid

$L=$ length of tube

$r=$ radius of tube

Equation 1 shows how nasal airway resistance is inversely proportional to the fourth power of the radius, now shown in equation 2:

$$
\text { Resistance } \propto \frac{1}{\mathbf{r}^{4}}
$$

We also know that the cross-sectional area of the nasal cavity, which is approximately cylindrical, is given by $\pi r^{2}$, such that the cross-sectional area is proportional to the square of its radius, now shown in equation 3 :

$$
\text { Area } \propto r^{2}
$$

Combining the two relationships in equations 2 and 3, we can predict that resistance will then be inversely proportional to the square of the cross-sectional area as shown in equation 4:

$$
\text { Resistance } \propto \frac{1}{\text { Area }^{2}}
$$

Based on this relationship, the value of $M C A_{1}$ on each side was squared and its inverse was determined. This gives us a representative value of the nasal airway resistance for the nasal cavity on the corresponding side. The full list of airflow rates and resistance values can be found in appendix 1. 
Due to the varying demographics such as age (e.g. C001/C002/A010's was 23, C031's was 68), height (e.g. C002's was $1.48 \mathrm{~m}, \mathrm{C} 032$ 's was $1.83 \mathrm{~m}$ ) and ethnic backgrounds (62\% Caucasian, $18 \%$ as Asian, $10 \%$ as Black, $10 \%$ as Others), subjects had different maximum inspiratory efforts i.e. transnasal pressures which affected how the airflow rates and resistances relate to each other. These confounding variables would have to be accounted for before plotting a graph of $1 /$ Resistance against Airflow Rate. To overcome this problem, a ratio of right and left airflow rates was taken for each subject. This was repeated for the nasal airway resistances. Doing this allowed the transnasal pressure of every subject to be standardised. The following shows how transnasal pressure of every subject can be standardized by taking ratios of both airflow and resistance values sii: $^{\text {ii. }}$

Using Poiseuille's Law, let equation 5 describe airflow for the right nasal cavity:

$$
\Delta \mathbf{P}_{\mathbf{R}}=\mathbf{Q}_{\mathbf{R}} \mathbf{R}_{\mathbf{R}}
$$

Similarly, let equation 6 describe airflow for the left nasal cavity:

$$
\Delta \mathbf{P}_{\mathbf{L}}=\mathbf{Q}_{\mathbf{L}} \mathbf{R}_{\mathbf{L}}
$$

Divide equation 5 by equation 6 :

$$
\frac{\Delta \mathbf{P}_{\mathbf{R}}}{\Delta \mathbf{P}_{\mathbf{L}}}=\frac{\mathbf{Q}_{\mathbf{R}} \mathbf{R}_{\mathbf{R}}}{\mathbf{Q}_{\mathbf{L}} \mathbf{R}_{\mathbf{L}}}
$$

Assuming $\Delta \mathbf{P}_{\mathbf{L}}=\Delta \mathbf{P}_{\mathbf{R}}$, i.e. maximum inspiratory effort was the same for the unilateral right and left PNIF measurement, then equation 7 becomes:

$$
\mathbf{1}=\frac{\mathbf{Q}_{\mathbf{R}} \mathbf{R}_{\mathbf{R}}}{\mathbf{Q}_{\mathbf{L}} \mathbf{R}_{\mathbf{L}}}
$$

Rearranging equation 8 , such that resistance terms are on the left, and airflow rate terms are on the right, we obtain:

$$
\frac{\mathbf{R}_{\mathbf{L}}}{\mathbf{R}_{\mathbf{R}}}=\frac{\mathbf{Q}_{\mathbf{R}}}{\mathbf{Q}_{\mathbf{L}}}
$$

Now, if we define the airflow rate ratio $\left(I^{\prime}\right)$ as $\frac{\mathbf{Q}_{R}}{\mathbf{Q}_{L}}$, and resistance ratio $\left(R^{\prime}\right)$ as $\frac{\mathbf{R}_{R}}{\mathbf{R}_{L}}$, equation 9 then becomes:

$$
\frac{\mathbf{1}}{\mathbf{R}^{\prime}}=\mathbf{I}^{\prime}
$$

\footnotetext{
iii The subscript ' $R$ ' and ' $L$ ' represent the variable for the right and left nasal cavity respectively.
} 
Equation 10 implies that plotting a graph of $1 /$ resistance ratio against airflow rate ratio will yield a directly proportional relationship, even if subjects had varying maximum inspiratory efforts. The full list of airflow rate and resistance ratios can be found in appendix 2. 


\section{RESULTS}

Following the exclusion of 3 control participants owing to a reduced olfaction, a regressed line of $1 /$ Resistance Ratio against Airflow Rate Ratio was plotted with the remaining 36 subjects as shown in figure 1. Our regressed line demonstrates a correlation coefficient of $r=0.76$, indicating 1/Resistance Ratio strongly correlates with the Airflow Rate Ratio, with a p-value of $<0.01$. This is in line with our hypothesis that data points of controls with a normal nasal cycle follow this linear relationship and lie closely along the regressed line.

Based on the same regressed line, figure 2 focuses on subjects with mucosal nasal congestion and how a threshold value could help to differentiate between congestion caused by inflammation (i.e. rhinitis) and that caused by the nasal cycle. Interestingly, olfaction seems to be a sensitive discriminator, and subjects found to have abnormal olfaction are highlighted lying outside of the threshold lines.

On the other hand, figure 3 focuses on patients with structural nasal obstruction of the internal nasal and how a threshold value could help to differentiate between an anatomical variation and pathology. Some of these subjects are highlighted lying outside of the threshold lines.

The concept of the threshold lines is explored further in the Discussion section. 


\section{DISCUSSION}

\section{Synopsis of key findings}

In this study, we have demonstrated a linear relationship between 1/Resistance Ratio and Airflow Rate Ratio based on empirical unilateral PNIF and AR data without having used nasal decongestants. This enables us to measure the nasal cycle. Data points of normal subjects with an ongoing active nasal cycle will move along the line. The extent of movement depends at which stage of the nasal cycle the subject happens to be in when the measurements are performed. Additionally, it can help predict both inflammation in the form of rhinitis as well as structural abnormalities such as septal deviation and nasal valve dysfunction.

- Predicting Rhinitis in Patients

Due to nasal congestion, air turbulence increases and a deviation from the model will occur. We believe that a slight deviation from the model exists due to physiological causes such as the nasal cycle, however, a more severe deviation from the model would be caused by pathological conditions such as mucosal inflammation. Rhinitis as an inflammatory condition causes more turbulence than that caused by the nasal cycle $[9,10]$.

Assuming this is true, there should be an upper limit of deviation imposed, such that deviation below this limit would be accepted as being due to the nasal cycle, whilst deviation above this limit would be taken to be due to rhinitis. As demonstrated in figure 2 , the threshold lines are set parallel to the regressed line on either side as turbulence is postulated to cause deviation from it. The upper and lower thresholds, indicated by the dashed orange and blue lines respectively, are prediction intervals set 3 SDs above and below the predicted regressed line.

Due to the small number of subjects who have olfactory dysfunction, it is difficult to determine how sensitive or specific our threshold values are. Larger sample sizes are required in future studies to know where exactly the upper limits should be imposed to ensure a high sensitivity and specificity for this model.

- Predicting Pathological Variations in the Internal Nasal Valve

In line with theoretical expectations, our results show that controls have roughly the same $\mathrm{MCA}_{1}$ on both sides, giving them a 1 /Resistance Ratio of approximately 1 . In more extreme cases whereby the $1 /$ Resistance Ratio of data points was much higher or lower than 1, these data points belonged to patients (such as A003, A008 and A018 on the top right extreme, and A007, A010, A011 and A015 on the bottom left extreme) with a pathological variation in the internal structure of the nose such as a septal deviation. Subjects with 1/Resistance Ratio measurements significantly away from 1 are unlikely to be due to just anatomical variations that are considered within normal limits. 
Hence, by using the same threshold concept as used for predicting rhinitis, pathological variations of the internal nasal valve such as unilateral internal valve collapse, septal deviation, or previous trauma could also be predicted. The threshold lines are set perpendicular to the $y-$ axis i.e. $1 /$ Resistance Ratio since any subjects above or below have $1 /$ Resistance Ratio values significantly away from 1 , which indicates a large difference in $\mathrm{MCA}_{1}$ values between either side. These likely belong to patients who have pathological variation of the internal nasal valve. These thresholds do not relate to the regressed line per se, but the graph can still be used to identify patients with a large difference in $\mathrm{MCA}_{1}$ values between either side, regardless of the airflow rate ratios. Thresholds were set at 1 SD above and below the mean of 1 /resistance ratio using orange and blue dashed lines respectively to divide the graph into the 'anatomical variation' or 'pathological variation' zone. Any deviation over and above this would be considered a pathological variation. This is illustrated in figure 3.

Based on the threshold values we have set for this model, we hypothesise the findings would exhibit high specificity because only patients are found outside of the threshold lines, but moderate sensitivity, as there are still patients who are found within the threshold lines. Once again, how exactly the threshold between anatomical and pathological variation to get best sensitivity and specificity requires further study.

\section{Limitations}

In this model, it was assumed the inspiratory effort for the left UPNIF reading was equal to that of the right. Even though this is relatively valid, it must be noted that the two readings were still separately taken, albeit one after the other, and the maximum inspiratory effort for each side could have differed slightly for the same subject.

Additionally, although AR can accurately determine $\mathrm{MCA}_{1}[13]$, simply taking the inverse of the square of the $\mathrm{MCA}_{1}$ to obtain a resistance value is simplified. This method of calculating resistance can be applied to uniform cylinders, which does not necessarily apply to the nasal cavity whose cross-sectional area varies continuously. A better way would have been to integrate the cross-sectional area along the nasal passage and use the result as an indicator of resistance.

\section{Clinical Applicability of the Study}

This model could further enable the clinician to make a nasal blockage diagnosis of either rhinitis or a deviated nasal septum or a combination of both and help plan treatment strategies as well as improve treatment monitoring. Equally, there is an increasing need for an objective nasal device which can correlate better with the patient's nasal symptomology and quality of life and importantly allow the patient to be better understand their condition[14-16]. Despite the high burden of nasal blockage, its diagnosis and management remains challenging[17].

\section{Future Study}


This model will be further developed to account for measurement errors and turbulent flow among other factors. Additionally, the nasal cycle could be measured in controls and patients multiple times longitudinally. Successive UPNIF and UMCA values of each subject could then be plotted to observe trends in the nasal cycle and perhaps be co-related with subjective symptoms at each measurement. 


\section{CONCLUSION}

A linear relationship between the $1 /$ Resistance Ratio and Airflow Rate Ratio using preliminary empirical UPNIF and UMCA data was demonstrated in our control group without the use of decongestants. This suggests data points of controls with a normal nasal cycle lie closely along the regressed line and equally those data points which lie outside the regressed line will help predict structural and inflammatory causes for nasal obstruction. 


\section{REFERENCES}

[1] Kayser R. Die exakte Messung der Luftdurchgängigkeit der Nase. Arch. Laryng. Rhinol. (Berlin) 1895;8:101

[2] Pendolino A, Lund V, Nardello E, Ottaviano G. The nasal cycle: a comprehensive review. Rhinology Online. 2018;1:67-76. doi:10.4193/RHINOL/18.021

[3] Pendolino A, Nardello E, Lund V et al. Comparison between unilateral PNIF and rhinomanometry in the evaluation of nasal cycle. Rhinology journal. 2018;56(2):122-126. doi:10.4193/rhin17.168

[4] GUNGOR, A., MOINUDDIN, R., NELSON, R., \& COREY, J. (1999). Detection of the Nasal Cycle with Acoustic Rhinometry. Otolaryngology-Head And Neck Surgery, 120(2), 238247. doi: 10.1016/s0194-5998(99)70413-4

[5] Carney A, Bateman N, Jones N. Reliable and reproducible anterior active rhinomanometry for the assessment of unilateral nasal resistance. Clin Otolaryngol Allied Sci. 2000;25(6):499-503. doi:10.1046/j.1365-2273.2000.00384.x

[6] Seifter J, Ratner A, Sloane D. Concepts In Medical Physiology. 1st ed. Philadelphia, Pa: Lipincott Williams \& Wilkins; 2005:249.

[7] Raeburn D, Giembycz M. Airways Smooth Muscle: Modelling The Asthmatic Response In Vivo. Basel: Birkhäuser Basel; 1996.

[8] Önerci, T. (2013). Nasal Physiology and Pathophysiology of Nasal Disorders (p. 366). Berlin, Heidelberg: Springer Berlin Heidelberg.

[9] Bende M, Barrow I, Heptonstall J et al. Changes in Human Nasal Mucosa during Experimental Coronavirus Common Colds. Acta Oto-Laryngologica. 1989;107(3-4):262-269. doi:10.3109/00016488909127507.

[10] Flint P, Cummings C, Phelps T. Cummings Otolaryngology Head \& Neck Surgery. 6th ed. Philadelphia: Mosby/Elsevier; 2010:617

[11] Rohrich R, Adams W, Ahmad J, Gunter J. Dallas Rhinoplasty. 1st ed. Hoboken: CRC Press; 2014:33-34.

[12] Fisher E, Lund V, Scadding G. Acoustic rhinometry in rhinological practice: discussion paper. Journal of the Royal Society of Medicine. 1994;87:411-413. 
[13] Cakmak O, Tarhan E, Coskun M, Cankurtaran M, Çelik H. Acoustic Rhinometry: Accuracy and Ability to Detect Changes in Passage Area at Different Locations in the Nasal Cavity. Annals of Otology, Rhinology \& Laryngology. 2005;114(12):949-957. doi:10.1177/000348940511401211.

[14] Andrews P, Choudhury N, Takhar A, Poirrier A, Jacques T, Randhawa $P$. The need for an objective measure in septorhinoplasty surgery: are we any closer to finding an answer?. Clinical Otolaryngology. 2015;40(6):698-703. doi:10.1111/coa.12455.

[15] Poirrier A, Ahluwalia S, Goodson A, Ellis M, Bentley M, Andrews P. Is the Sino-Nasal Outcome Test-22 a suitable evaluation for septorhinoplasty?. The Laryngoscope. 2012;123(1):76-81. doi:10.1002/lary.23615.

[16] Whitcroft K, Andrews P, Randhawa P. Peak nasal inspiratory flow correlates with quality of life in functional endoscopic sinus surgery. Clinical Otolaryngology. 2017:1-6. doi:10.1111/coa.12859.

[17] Andrews P, Joseph J, Li C, Nip L, Jacques T, Leung T. A UK survey of current ENT practice in the assessment of nasal patency. The Journal of Laryngology \& Otology. 2017;131(08):702706. doi:10.1017/s0022215117001311.

\section{List of Titles and Captions of Figures}

Figure 1: Graph of 1/Resistance against Airflow Rate Ratio of the remaining 36 subjects, after removing 3 outliers (C023, C026 and C033) due to poor olfaction

Figure 2: Graph of 1/Resistance Ratio against Airflow Rate Ratio, with upper and lower limits demarcating deviation deemed to be caused by nasal cycle

Figure 3: Graph of 1/Resistance Ratio against Airflow Rate Ratio, with the cut-off limits demarcating the boundary between the 'anatomical variation' zone and 'pathological variation' zone 
Appendices $^{\mathrm{i}}$

Appendix 1

Table showing the airflow rate and airway resistance measurements of all controls and patients on each side:

\begin{tabular}{|c|c|c|c|c|}
\hline \multirow{2}{*}{$\begin{array}{c}\text { Subject } \\
\text { ID }\end{array}$} & \multicolumn{2}{|c|}{ Airflow Rate } & \multicolumn{2}{|c|}{ Resistance } \\
\hline & Right/L min-1 & Left/L min $^{-1}$ & Right $/ \mathrm{cm}^{-4}$ & Left $/ \mathrm{cm}^{-4}$ \\
\hline C001 & 50 & 50 & 4.64 & 6.22 \\
\hline $\mathrm{COO2}$ & 60 & 80 & 1.83 & 2.50 \\
\hline $\mathrm{COO4}$ & 60 & 80 & 2.48 & 2.66 \\
\hline C005 & 70 & 60 & 4.71 & 2.35 \\
\hline C006 & 80 & 80 & 2.22 & 2.16 \\
\hline $\mathrm{COO7}$ & 70 & 50 & 3.18 & 3.35 \\
\hline C008 & 100 & 40 & 0.80 & 1.56 \\
\hline CO09 & 70 & 100 & 10.96 & 3.61 \\
\hline C010 & 60 & 60 & 11.26 & 5.28 \\
\hline C011 & 60 & 40 & 6.44 & 7.04 \\
\hline $\mathrm{C} 013$ & 110 & 115 & 4.53 & 3.84 \\
\hline C014 & 70 & 90 & 6.64 & 7.42 \\
\hline C016 & 120 & 100 & 4.16 & 2.98 \\
\hline C017 & 40 & 50 & 3.18 & 2.53 \\
\hline $\mathrm{C} 018$ & 100 & 110 & 3.29 & 3.83 \\
\hline C019 & 130 & 100 & 1.81 & 1.32 \\
\hline $\mathrm{CO} 22$ & 90 & 90 & 3.71 & 3.48 \\
\hline $\mathrm{CO23}$ & 50 & 60 & 1.27 & 2.28 \\
\hline $\mathrm{CO} 24$ & 100 & 60 & 2.67 & 4.73 \\
\hline $\mathrm{CO25}$ & 100 & 100 & 5.62 & 9.35 \\
\hline $\mathrm{CO26}$ & 140 & 80 & 4.15 & 1.67 \\
\hline $\mathrm{CO27}$ & 100 & 100 & 2.41 & 1.32 \\
\hline $\mathrm{CO28}$ & 70 & 40 & 4.30 & 6.16 \\
\hline CO29 & 45 & 50 & 1.40 & 2.27 \\
\hline C031 & 70 & 40 & 2.06 & 4.02 \\
\hline $\mathrm{CO33}$ & 45 & 65 & 1.30 & 12.57 \\
\hline
\end{tabular}




\begin{tabular}{|l|c|c|c|c|}
\hline $\mathrm{A} 001$ & 50 & 50 & 2.88 & 2.29 \\
\hline $\mathrm{A} 002$ & 60 & 80 & 1.08 & 1.93 \\
\hline $\mathrm{A} 003$ & 90 & 40 & 3.08 & 7.15 \\
\hline $\mathrm{A} 004$ & 60 & 80 & 10.21 & 15.75 \\
\hline $\mathrm{A} 007$ & 70 & 50 & 10.34 & 0.60 \\
\hline $\mathrm{A} 008$ & 100 & 40 & 1.71 & 4.27 \\
\hline $\mathrm{A} 009$ & 70 & 100 & 11.89 & 4.41 \\
\hline $\mathrm{A} 010$ & 60 & 60 & 4.43 & 1.16 \\
\hline $\mathrm{A} 011$ & 60 & 40 & 5.51 & 0.78 \\
\hline $\mathrm{A} 012$ & 40 & 45 & 0.81 & 1.00 \\
\hline $\mathrm{A} 015$ & 120 & 80 & 4.34 & 1.28 \\
\hline $\mathrm{A} 016$ & 120 & 100 & 20.66 & 39.06 \\
\hline $\mathrm{A} 018$ & 100 & 110 & 1.71 & 3.52 \\
\hline
\end{tabular}

Appendix 2

Table showing the airflow rate ratios and airway resistance ratios of all controls and patients:

\begin{tabular}{|c|c|c|}
\hline Subject ID & Airflow Rate Ratio & Resistance Ratio \\
\hline C001 & 1.00 & 0.75 \\
\hline C002 & 0.75 & 0.73 \\
\hline C004 & 0.75 & 0.93 \\
\hline C005 & 1.17 & 2.00 \\
\hline C006 & 1.00 & 1.03 \\
\hline C007 & 1.40 & 0.95 \\
\hline C008 & 2.50 & 0.51 \\
\hline C009 & 0.70 & 3.03 \\
\hline C010 & 1.00 & 2.13 \\
\hline C011 & 1.50 & 0.92 \\
\hline C013 & 0.96 & 1.18 \\
\hline C014 & 0.78 & 0.89 \\
\hline C016 & 1.20 & 1.40 \\
\hline C017 & 0.80 & 1.26 \\
\hline C018 & 0.91 & 0.86 \\
\hline
\end{tabular}




\begin{tabular}{|c|c|c|}
\hline C019 & 1.30 & 1.37 \\
\hline $\mathrm{CO} 22$ & 1.00 & 1.07 \\
\hline C023 & 0.83 & 0.56 \\
\hline $\mathrm{CO24}$ & 1.67 & 0.56 \\
\hline CO25 & 1.00 & 0.60 \\
\hline C026 & 1.75 & 2.48 \\
\hline $\mathrm{CO} 27$ & 1.00 & 1.82 \\
\hline $\mathrm{CO} 28$ & 1.75 & 0.70 \\
\hline CO29 & 0.90 & 0.62 \\
\hline C031 & 1.75 & 0.51 \\
\hline C033 & 0.69 & 0.10 \\
\hline A001 & 1.17 & 1.26 \\
\hline A002 & 2.17 & 0.56 \\
\hline A003 & 2.25 & 0.43 \\
\hline A004 & 1.80 & 0.65 \\
\hline A007 & 0.33 & 17.21 \\
\hline A008 & 2.33 & 0.40 \\
\hline A009 & 1.00 & 2.69 \\
\hline A010 & 0.80 & 3.82 \\
\hline A011 & 0.55 & 7.04 \\
\hline A012 & 0.89 & 0.81 \\
\hline A015 & 0.50 & 3.38 \\
\hline A016 & 0.86 & 0.53 \\
\hline A018 & 2.67 & 0.49 \\
\hline
\end{tabular}




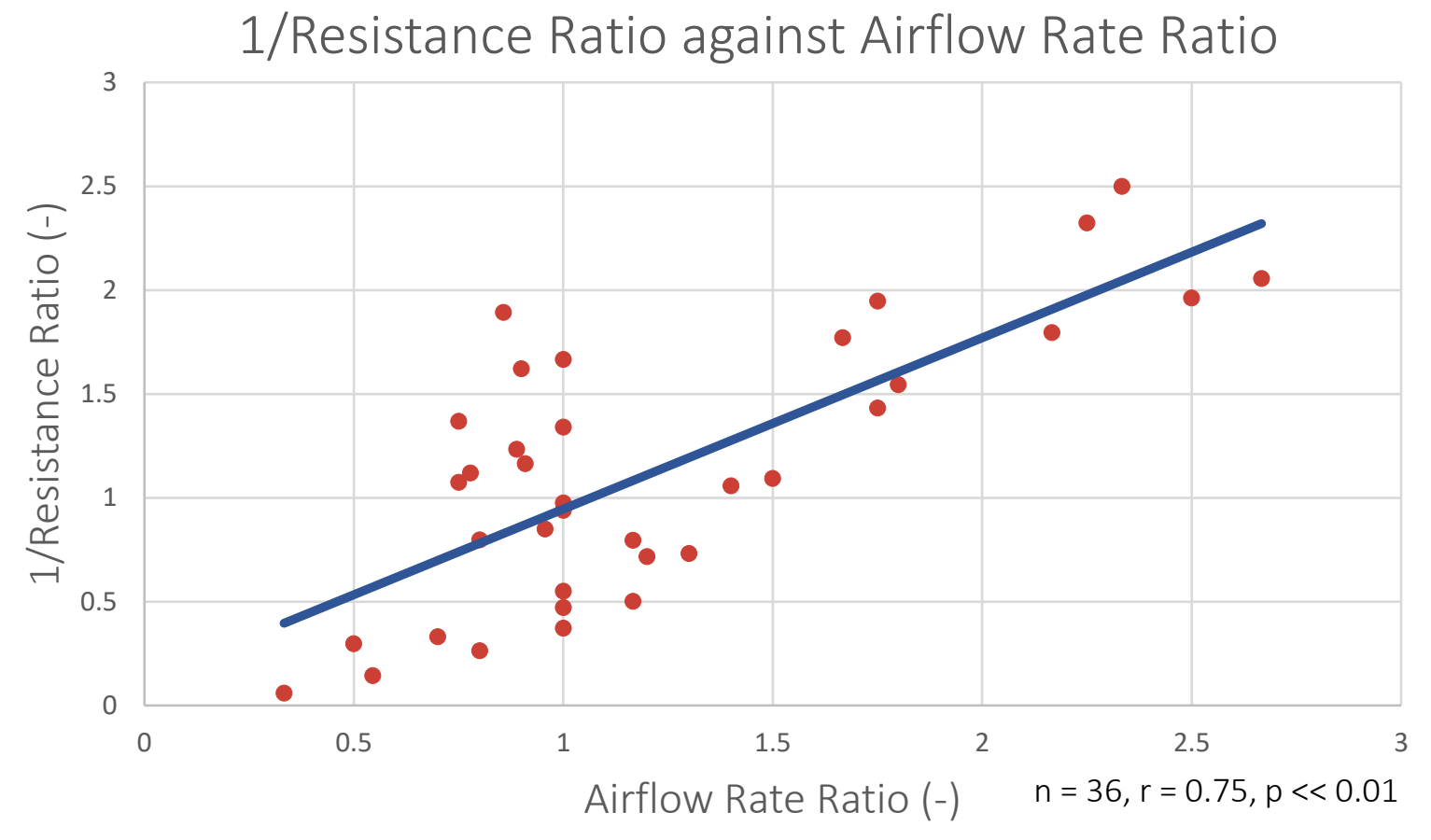

Figure 1: Graph of 1/Resistance against Airflow Rate Ratio of the remaining 36 subjects, after removing 3 outliers (CO23, CO26 and C033) due to poor olfaction 


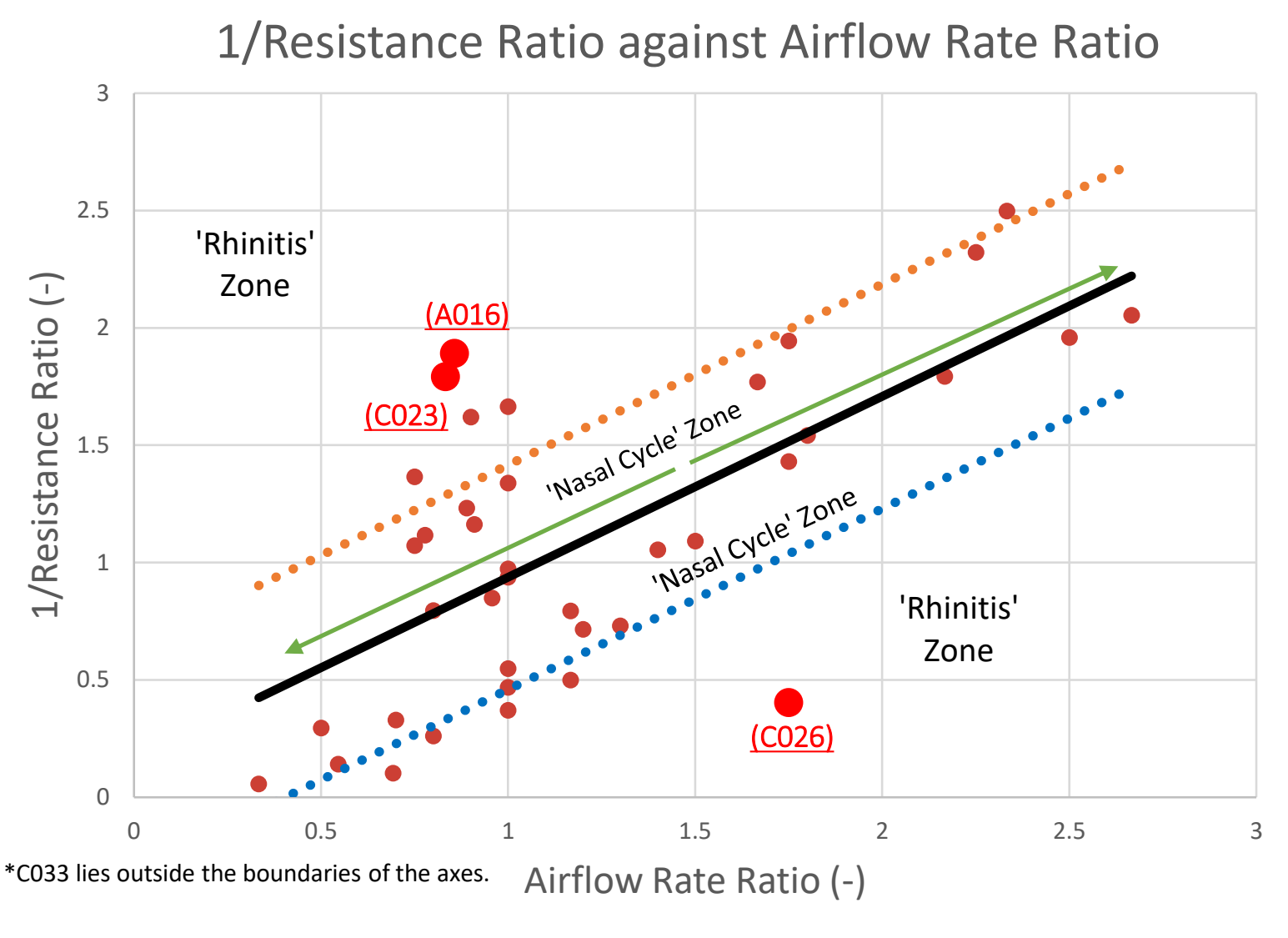

Figure 2: Graph of 1/Resistance Ratio against Airflow Rate Ratio, with upper and lower limits demarcating deviation deemed to be caused by the nasal cycle. 


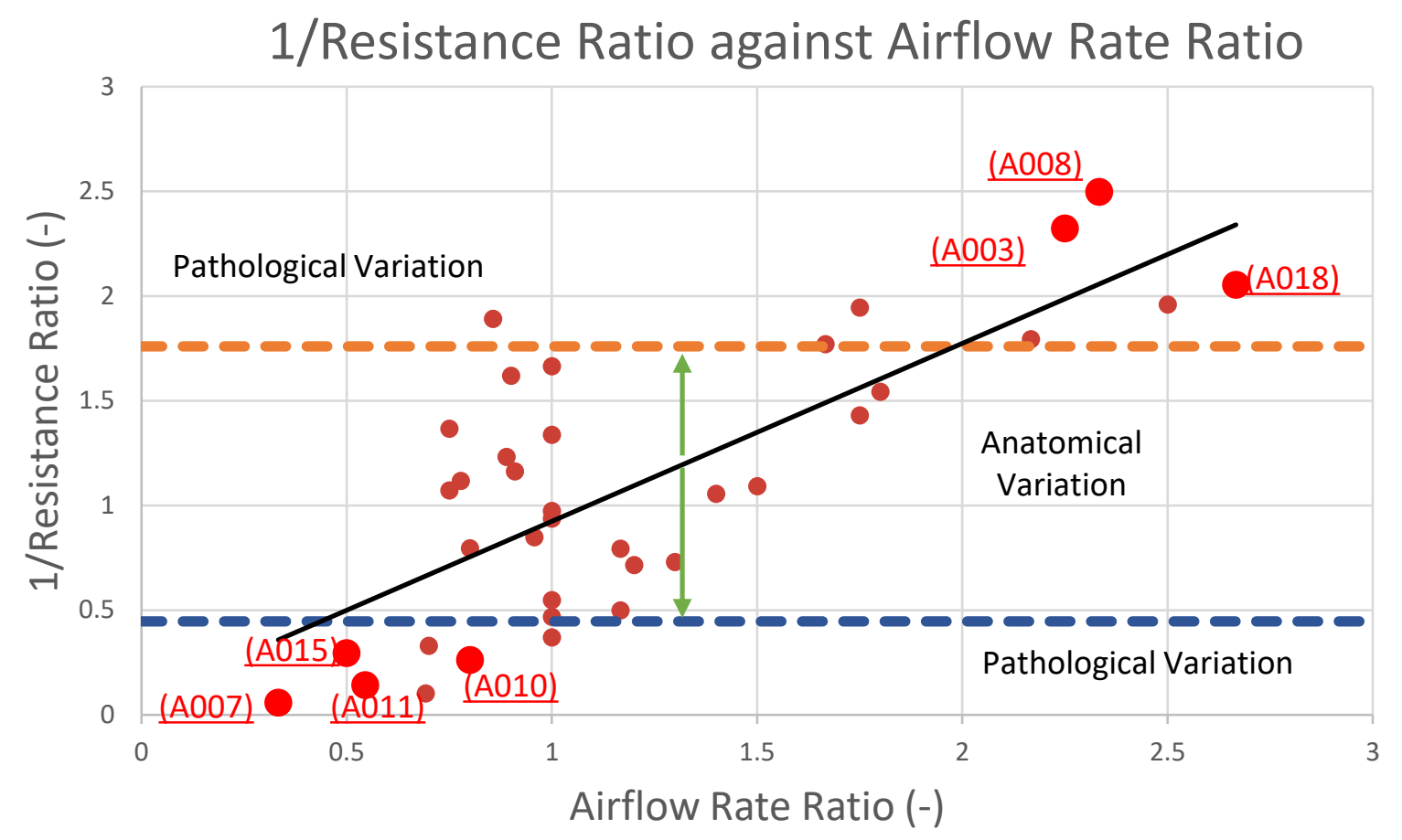

Figure 3: Graph of 1/Resistance Ratio against Airflow Rate Ratio, with the cut-off limits demarcating the boundary between the 'anatomical variation' zone and 'pathological variation' zone 